\title{
Tooth-shaped plasmonic waveguide filters with nanometeric
}

\section{sizes}

\author{
Xian-Shi LIN and Xu-Guang HUANG* \\ Laboratory of Photonic Information Technology, South China Normal University, Guangzhou, \\ 510006, China \\ *Corresponding author: huangxg@scnu.edu.cn
}

\begin{abstract}
A novel nanometeric plasmonic filter in a tooth-shaped Metal-Insulator-Metal waveguide is proposed and demonstrated numerically. An analytic model based on the scattering matrix method is given. The result reveals that the single tooth-shaped filter has a wavelength filtering characteristic and an ultra-compact size in the length of a few hundred nanometers, compared to grating-like SPPs filters. Both analytic and simulation results show that the wavelength of the trough of the transmission has linear and nonlinear relationships with the tooth depth and the tooth width, respectively. The waveguide filter could be utilized to develop ultra-compact photonic filters for high integration. (C) 2008 Optical Society of America
\end{abstract}

OCIS codes: $130.3120,230.7408,240.6680,290.5825$. 
Surface Plasmons are waves that propagate along a metal-dielectric interface with an exponentially decaying field in the both sides $[1,2]$. The unique properties of Surface Plasmon Plolaritons (SPPs) have shown the potential to overcome the diffraction limit in conventional optics, which could be utilized to achieve nano-scale photonic devices for high integration. Several different Metal-Insulator-Metal (MIM) waveguide structures based on SPPs have been numerically and/or experimentally demonstrated such as U-shaped waveguides [3], splitters [4], Y-shaped combiner [5], Multimode-Interferometers [6], couplers [7,8], M-Z interferometers [9,10], Bragg mirrors [11], and photonic bandgap structures [12]. To achieve wavelength filtering characteristics, SPPs Bragg reflectors and nanocavities have been proposed. They include the metal heterostructures constructed with several periodic slots vertically along a MIM waveguide [13,14], the Bragg grating fabricated by periodic modulating the thickness of thin metal stripes embedded in a insulator [15] and the periodic structure formed by changing alternately two kinds of the insulators with the same width [16] or different widths $[17,18]$. Lately, a high-order plasmonic Bragg reflector with a periodic modulation of the core index of the insulators [19], and a structure with periodic variation of the width of the insulator in MIM waveguide [20] have been proposed. Most of the structures mentioned above, however, have the period number of $\mathrm{N}>9$ with the total lengths over $4 \mu \mathrm{m}$ and beyond subwavelength-scale, which result in relatively high insertion loss of several $\mathrm{dB}$. In this letter, a nanoscale SPPs filter based on the MIM waveguide consisting of single rectangular tooth is proposed. The SPPs distributions and propagations are characterized by the method of Finite-Difference Time-Domain (FDTD), with perfect-matching-layer absorbing boundary conditions. An analytic model based on the scattering matrix method is derived to explain the filtering mechanism of the structure. 
To begin with the dispersion relation of the fundamental TM mode in a MIM waveguide (shown in the inset of Fig. 1) is given by $[15,21]$ :

$$
\varepsilon_{i n} k_{z 2}+\varepsilon_{m} k_{z 1} \operatorname{coth}\left(-\frac{i k_{z 1}}{2} w\right)=0
$$

with $k_{\mathrm{z} 1}$ and $k_{\mathrm{z} 2}$ defined by momentum conservations:

$$
k_{z 1}^{2}=\varepsilon_{i n} k_{0}^{2}-\beta^{2}, \quad k_{z 2}^{2}=\varepsilon_{m} k_{0}^{2}-\beta^{2} .
$$

Where $\varepsilon_{i n}$ and $\varepsilon_{m}$ are respectively dielectric constants of the insulator and the metal, $k_{0}=2 \pi / \lambda_{0}$ is the free-space wave vector. The propagation constant $\beta$ is represented as the effective index $n_{\text {eff }}$ $=\beta / k_{0}$ of the waveguide for SPPs. The real part of $n_{\text {eff }}$ of the slit waveguide as a function of the slit width at different wavelengths is shown in Fig. 1. It should be noted that the dependence of $n_{\text {eff }}$ on waveguide width is also suitable to the small part or region of the tooth waveguide with the tooth width of $w_{t}$ shown in Fig. 2. The imaginary part of $n_{\text {eff }}$ is referred to the propagation length which is defined as the length over which the power carried by the wave decays to 1/e of its initial value: $L_{\text {spps }}=\lambda_{0} /\left[4 \pi \cdot \operatorname{Im}\left(n_{\text {eff }}\right)\right]$. In the calculation above and the following simulations, the insulator in all of the structures is assumed to be air $\left(\varepsilon_{i n}=1\right)$, and the frequency-dependent complex relative permittivity of silver is characterized by Drude model: $\varepsilon_{m}(\omega)=\varepsilon_{\infty}-\omega_{p}^{2} / \omega(\omega+i \gamma)$. Here $\omega_{p}=1.38 \times 10^{16} \mathrm{~Hz}$ is the bulk plasma frequency, which represents the natural frequency of the oscillations of free conduction electrons. $\gamma=2.73 \times 10^{13} \mathrm{~Hz}$ is the damping frequency of the oscillations, $\omega$ is the angular frequency of the incident electromagnetic radiation, $\varepsilon_{\infty}$ stands for the dielectric constant at infinite angular frequency with the value of 3.7 [20].

The tooth-shaped waveguide filter is shown in Fig. 2. In the following FDTD simulations, the grid sizes in the $x$ and $z$ directions are chosen to be $5 \mathrm{~nm} \times 5 \mathrm{~nm}$. The fundamental TM mode of 
the plasmonic waveguide is excited by a dipole source. Two power monitors are respectively set at the points of $P$ and $Q$ to detect the incident and transmission fields for calculating the incident power of $P_{\text {in }}$ and the transmitted power of $P_{\text {out }}$. The transmittance is defined to be $T=P_{\text {out }} / P_{\text {in }}$. The length of $L$ is fixed to be $300 \mathrm{~nm}$, while the tooth width and depth are respectively $w_{t}=50 \mathrm{~nm}$ and $d=100 \mathrm{~nm}$. As shown in Fig. 3(a), the tooth-shaped waveguide is of a filtering function: A trough occurs at the free space wavelength nearly $784 \mathrm{~nm}$ with the transmittance of $\sim 0 \%$. The maximum transmittance at the wavelengths longer than $1700 \mathrm{~nm}$ is over $90 \%$. The contour profiles of the field distributions around the tooth-shaped area at different wavelengths are shown in Figs. 3(b)3(d). The filtering structure is distinguished from the Bragg reflectors based on periodical heterostructure.

The phenomenon above can be physically explained in the scattering matrix theory [23] as follows:

$$
\left(\begin{array}{l}
E_{1}^{\text {out }} \\
E_{2}^{\text {out }} \\
E_{3}^{\text {out }}
\end{array}\right)=S \cdot\left(\begin{array}{c}
E_{1}^{\text {in }} \\
E_{2}^{\text {in }} \\
E_{3}^{\text {in }}
\end{array}\right),
$$

where $S=\left[\begin{array}{lll}r_{1} & t_{1} & s_{3} \\ t_{1} & r_{1} & s_{3} \\ s_{1} & s_{1} & r_{3}\end{array}\right], r_{i}, t_{i}$ and $s_{i}(i=1,2,3)$ are respectively the reflection, transmission and splitting coefficients of a incident beam from Port $i(i=1,2,3)$, caused by the structure. $E_{\mathrm{i}}^{\mathrm{in}}$ and $E_{\mathrm{i}}^{\text {out }}$ stand for the fields of incident and output beams at Port $i$, respectively. Using the fact that $|S|=1$, one can obtain:

$$
r_{1}^{2} r_{3}+2 t_{1} s_{1} s_{3}-2 r_{1} s_{1} s_{3}-t_{1}^{2} r_{3}=1
$$


For the case of $E_{2}^{\text {in }}=0$, one has

$$
E_{2}^{\text {out }}=t_{1} E_{1}^{\text {in }}+s_{3} E_{3}^{\text {in }},
$$

in which $E_{3}^{\text {in }}$ is given as follows:

$$
E_{3}^{\text {in }}=s_{1} E_{1}^{\text {in }} \exp (i \phi(\lambda))\left(1+r_{3} \exp (i \phi(\lambda))+r_{3}^{2} \exp (2 i \phi(\lambda))+\ldots\right)=\frac{s_{1} E_{1}^{\text {in }}}{1-r_{3} \exp (i \phi(\lambda))} \exp (i \phi(\lambda)),
$$

where the phase delay $\phi(\lambda)=\frac{4 \pi}{\lambda} n_{\text {eff }} \cdot d+\Delta \varphi(\lambda)$, and $\Delta \varphi(\lambda)$ is the phase-shift caused by the reflection on the air-silver surface. Combined Eq. (5) and Eq. (6), the output field at Port 2 is derived as:

$$
E_{2}^{\text {out }}=t_{1} E_{1}^{\text {in }}+\frac{S_{1} S_{3} E_{1}^{\text {in }}}{1-r_{3} \exp (i \phi(\lambda))} \exp (i \phi(\lambda))
$$

Therefore, the transmittance $T$ from Port 1 to Port 2 is given by:

$$
T=\left|\frac{E_{2}^{\text {out }}}{E_{1}^{\text {in }}}\right|^{2}=\left|t_{1}+\frac{s_{1} s_{3}}{1-r_{3} \exp (i \phi(\lambda))} \exp (i \phi(\lambda))\right|^{2} .
$$

It can be seen from Eq. (8) that, if the phase satisfies $\phi(\lambda)=(2 m+1) \pi(m=0,1,2 \ldots)$, the two terms inside the absolute value sign on the right of the equation will cancel each other (as it can be seen in Fig. 3(c)), so that the transmittance $T$ will become minimum. Therefore, the wavelength $\lambda_{m}$ of the trough of transmission is determined as follows:

$$
\lambda_{m}=\frac{4 \cdot n_{e f f} \cdot d}{(2 m+1)-\frac{\Delta \varphi(\lambda)}{\pi}} .
$$


It can be seen that the wavelength $\lambda_{m}$ is linear to the tooth depth $d$, and depends on tooth width $w_{t}$, through the somewhat inverse-proportion-like relationship between $n_{\text {eff }}$ and $w_{t}$ shown in Fig. 1.

Figure 4(a) shows the transmission spectra of the waveguide filters with various tooth widths of $w_{t}$. The maximum transmittance can reach 97\%. Figure 4(b) shows the wavelength of the trough vs. the tooth width of $w_{t}$. The primary trough of the transmission moves very significantly to short wavelength (blue-shift) with the increase of $w_{t}$ for $w_{t}<20 \mathrm{~nm}$. The shift rate rapidly becomes small after $w_{t}>20 \mathrm{~nm}$, and tends to be saturated when $w_{t}>200 \mathrm{~nm}$. As revealed in the Eq. (9), the above relationship between the trough position and $w_{t}$ mainly results from the contribution of the inverse-proportion-like dependence of $n_{\text {eff }}$ on $w_{t}$. The change rate of $\Delta n_{\text {eff }} / \Delta w_{t}$ within the tooth width of $20 \mathrm{~nm}$ is much higher than that of $\Delta n_{\text {eff }} / \Delta w_{t}$ after $w_{t}>20 \mathrm{~nm}$, as shown in Fig. 1 and Ref.13, and becomes finally saturated after $w_{t}>200 \mathrm{~nm}$. Obviously, tooth width $w_{t}$ should be chosen within the range of $20-200 \mathrm{~nm}$ to avoid the critical behavior and the difficulty in fabrication process.

Figure 5(a) shows the transmission spectra of the filters with different tooth depths of $d$. It is found that the wavelength of the trough shifts to long wavelength with the increasing of $d$. Figure 5(b) reveals that the wavelength of the trough has a linear relationship with the tooth depth, as our expectation in Eq. (9). Therefore, one can realize the filter function in various required wavelength with high performance, by changing the width or/and the depth of the tooth. For example, to obtain a filter with a trough at the wavelength of $1550 \mathrm{~nm}$, the structural parameters of $w_{t}=w=50 \mathrm{~nm}$ and $d=237.5 \mathrm{~nm}$ can be chosen.

In summary, a novel plasmonic waveguide filter constructed with a MIM structure engraved single rectangular tooth is investigated. The filter is of an ultra-compact size with a few hundreds of nanometers in length, and then low insertion loss. Moreover, it is promised to reduce 
the difficulties in fabrication, comparing with previous grating-like heterostructures with a few micrometers in length. Our results suggest that the new structure could be utilized to develop plasmonic wavelength filters on flat metallic surfaces with extreme high integration for planar nanometeric photonic circuits.

The authors acknowledge the financial support from the Natural Science Foundation of Guangdong Province, China (Grant No. 07117866). 


\section{References}

1. H. Raether, Surface Plasmon on Smooth and Rough Surfaces and on Gratings (SpringerVerlag, Berlin, Germany, 1988).

2. W. L. Barnes, A. Dereux, and T. Ebbesen, "Surface plasmon subwavelength optics," Nature 424, 824-830 (2003).

3. T. Lee and S. Gray, "Subwavelength light bending by metal slit structures," Opt. Express 13, 9652-9659 (2005).

4. G. Veronis and S. Fan, "Bends and splitters in metal-dielectric-metal subwavelength plasmonic waveguides," Appl. Phys. Lett. 87, 131102 (2005).

5. H. Gao, H. Shi, C. Wang, C. Du, X. Luo, Q. Deng, Y. Lv, X. Lin, and H. Yao, "Surface plasmon polariton propagation and combination in Y-shaped metallic channels," Opt. Express 13, 10795-10800 (2005).

6. Z. Han and S. He, "Multimode interference effect in plasmonic subwavelength waveguides and an ultra-compact power splitter," Opt. Commun. 278, 199-203 (2007).

7. T. Nikolajsen, K. Leosson and S. I. Bozhevolnyia, "Surface plasmon polariton based modulators and switches operating at telecom wavelengths," Appl. Phys. Lett. 85, 5833 (2004).

8. H. Zhao, X. Huang and J. Huang, "Novel optical directional coupler based on surface plasmon polaritons," Phys. E 40, 3025-3029 (2008).

9. B. Wang and G. Wang, "Surface plasmon polariton propagation in nanoscale metal gap waveguides," Opt. Lett. 29, 1992-1994 (2004). 
10. Z. Han, L. Liu, and E. Forsberg, "Ultra-compact directional couplers and Mach-Zehnder interferometers employing surface plasmon polaritons," Opt. Commun. 259, 690-695 (2006).

11. H. Ditlbacher, J. R. Krenn, G. Schider, A. Leitner, and F. R. Aussenegg, "Two-dimensional optics with surface plasmon polaritons," Appl. Phys. Lett. 81, 1762 (2002).

12. S. I. Bozhevolnyi, J. E. Erland, K. Leosson, P. M. W. Skovgaard, and J. M. Hvam, "Waveguiding in surface plasmon polariton band gap structures," Phys. Rev. Lett. 86, 3008-3011 (2001).

13. B. Wang and G. Wang, "Plasmon Bragg reflectors and nanocavities on flat metallic surface," Appl. Phys. Lett. 87, 013107 (2005).

14. W. Lin and G. Wang, "Metal heterowaveguide superlattices for a plasmonic analog to electronic Bloch oscillations," Appl. Phys. Lett. 91, 143121 (2007).

15. A. Boltasseva, S. I. Bozhevolnyi, T. Nikolajsen, and K. Leosson, "Compact Bragg gratings for Long-Range surface plasmon polaritons," J. Lightwave Technol. 24, 912-918 (2006).

16. A. Hossieni and Y. Massoud, "A low-loss metal-insulator-metal plasmonic bragg reflector," Opt. Express 14, 11318-11323 (2006).

17. A. Hosseini, H. Nejati, and Y. Massoud, "Modeling and design methodology for metalinsulator-metal plasmonic Bragg reflectors," Opt. Express. 16, 1475-1480 (2008).

18. J. Q. Liu, L. L. Wang, M. D. He, W. Q. Huang, D. Y Wang, B. S. Zou, and S.C Wen, "A wide bandgap plasmonic Bragg reflector," Opt. Express. 16, 4888-4894 (2008).

19. J. Park, H. Kim, and B. Lee, "High order plasmonic Bragg reflection in the metal-insulatormetal waveguide Bragg grating," Opt. Express 16, 413-425 (2008). 
20. Z. Han, E. Forsberg, and S. He, "Surface plasmon Bragg gratings formed in metalinsulator-metal waveguides," IEEE Photon.Technol. Lett. 19, 91-93 (2007).

21. J. A. Dionne, L. A. Sweatlock, and H. A. Atwater, "Plasmon slot waveguides: Towards chip-scale propagation with subwavelength-scale localization," Phys. Rev. B 73, 035407 (2006).

22. E. D. Palik, Handbook of optical constants of solids (Academic Press, New York, NY 1985).

23. H. A. Haus, Waves and Fields in Optoelectronics (Prentice-Hall, Englewood Cliffs, NJ, 1984). 
Fig. 1

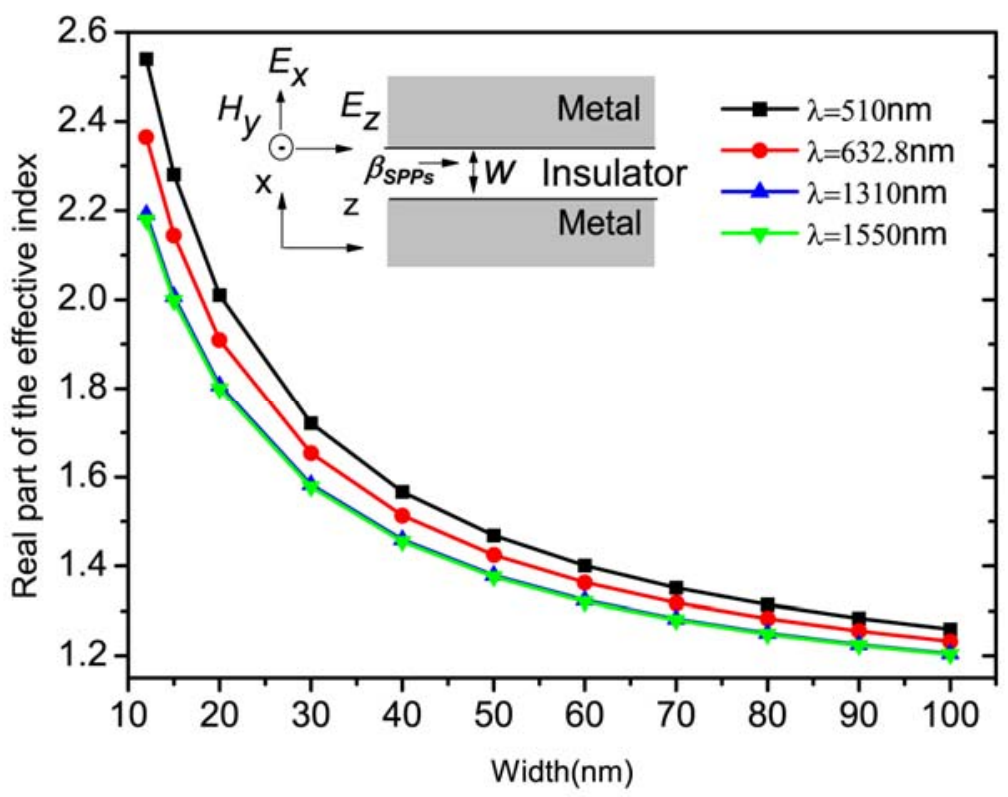


Fig. 2

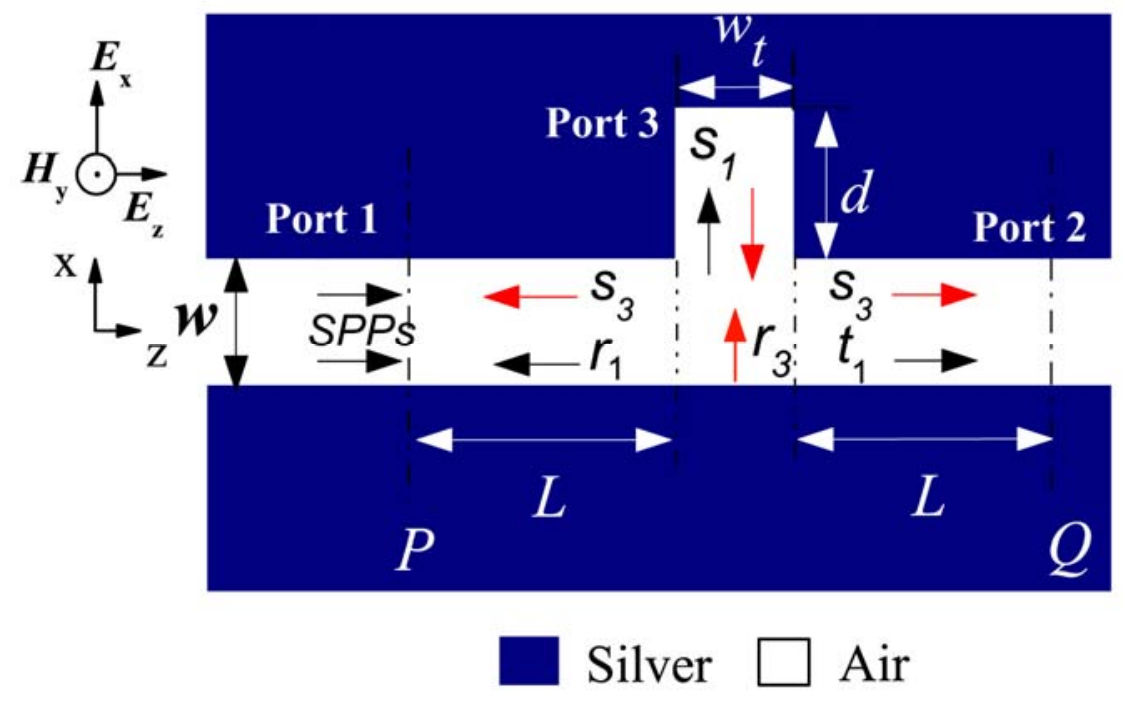


Fig. 3
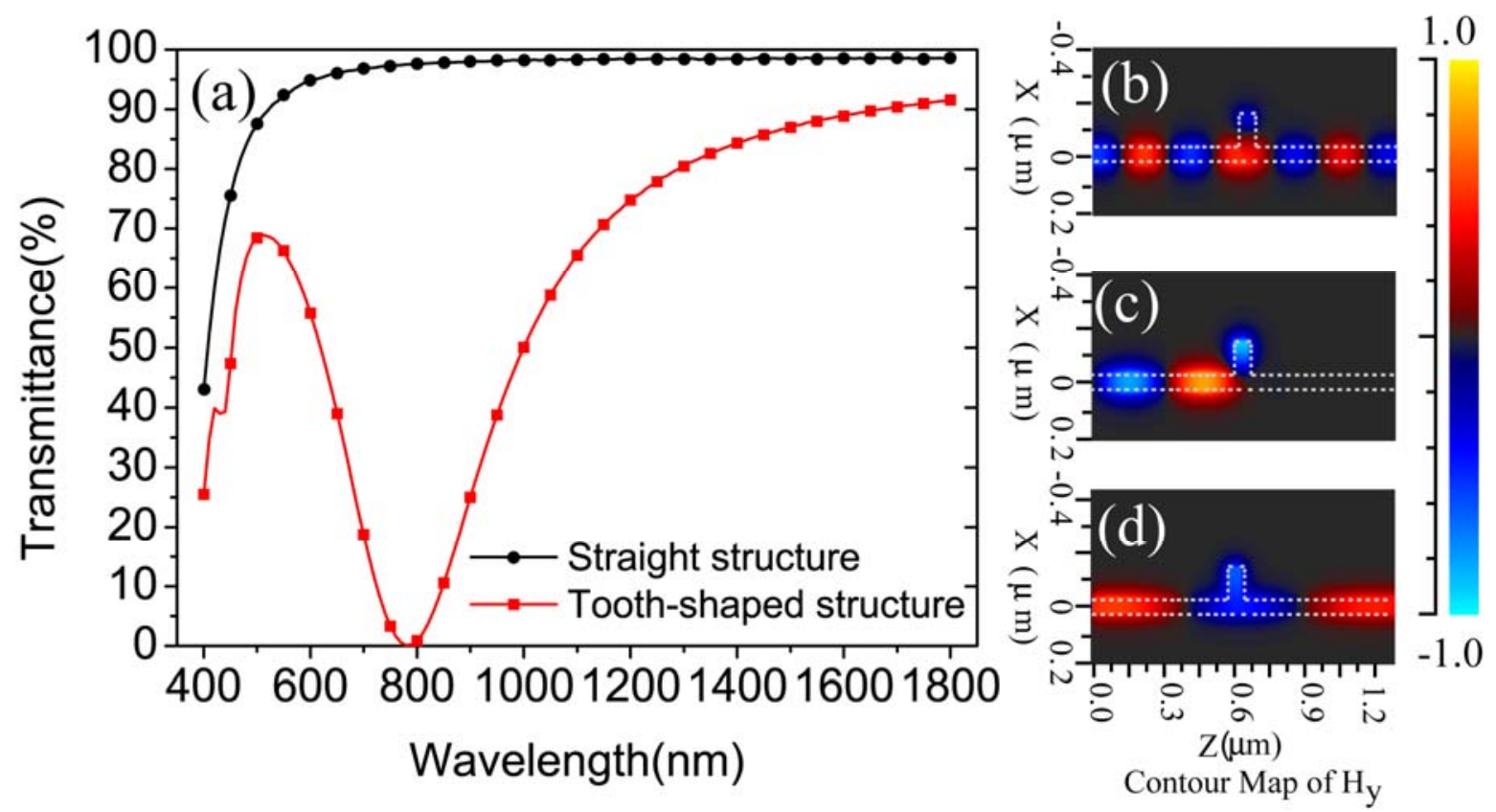
Fig. 4
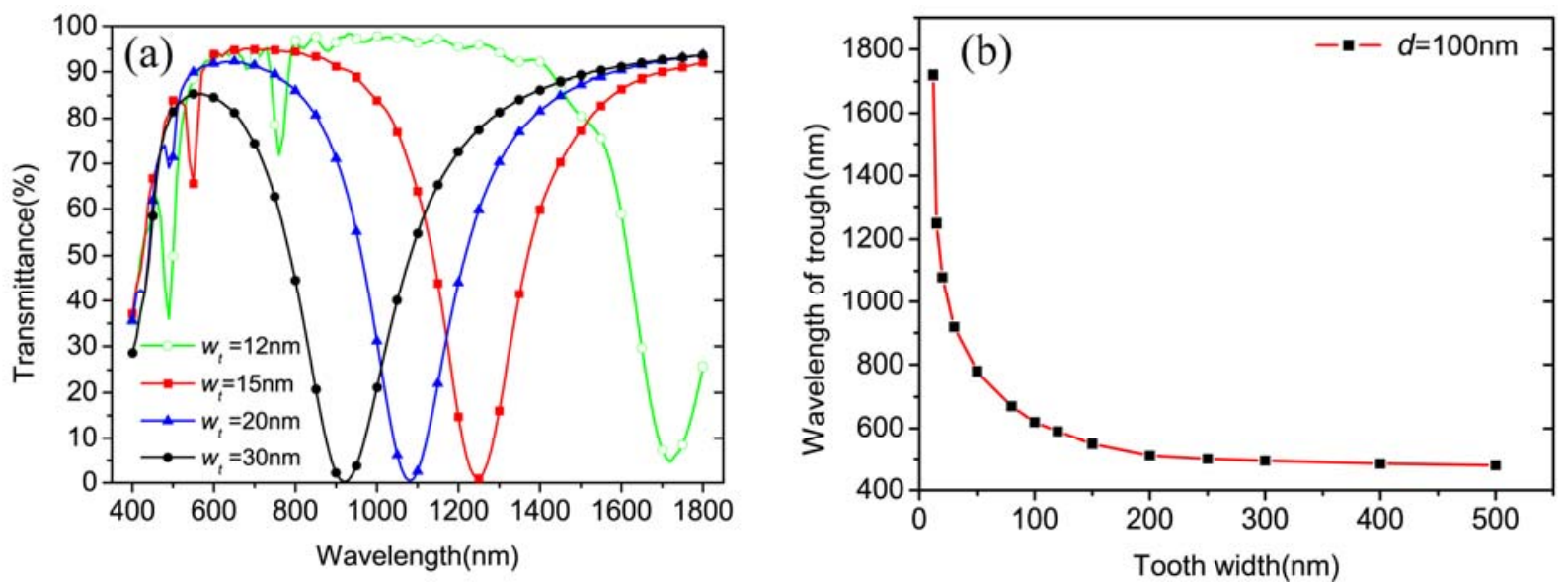
Fig. 5
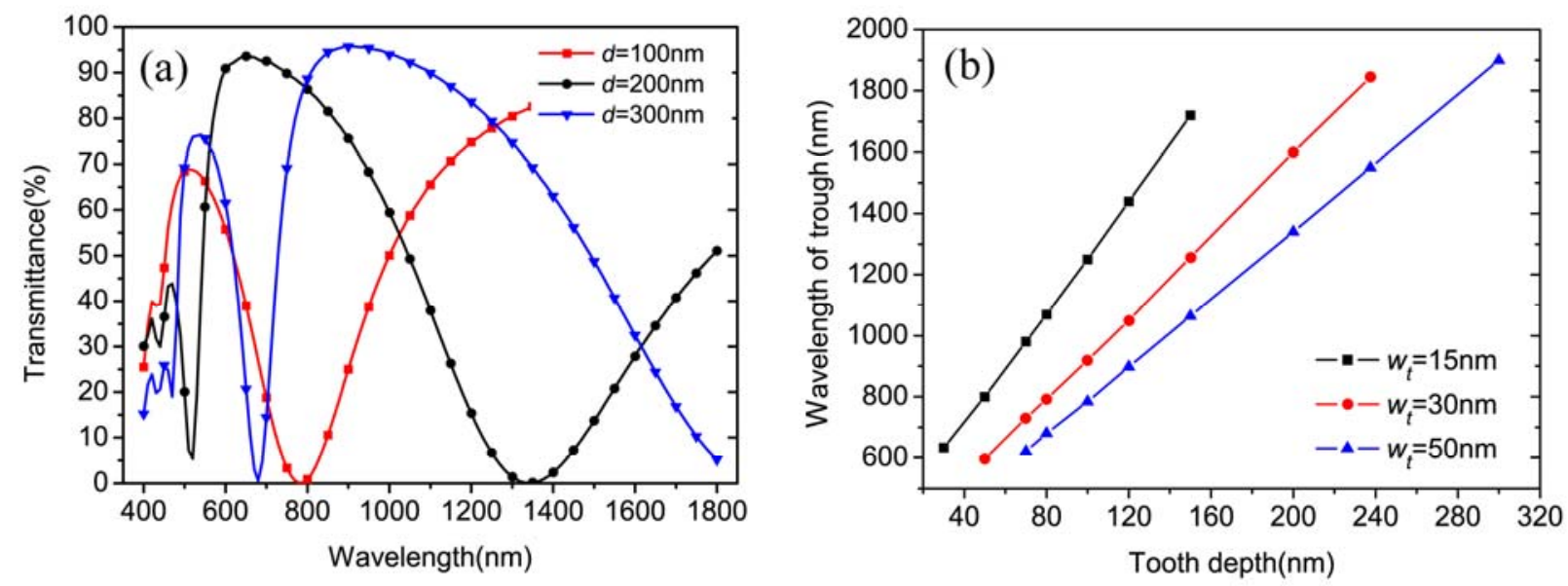


\section{Figure Captions}

Fig. 1. (Color online) Real part of the effective index of refraction versus the width of a MIM slit waveguide structure.

Fig. 2. (Color online) The structure schematics of a single tooth-shaped waveguide filter, with the slit width of $w$, the tooth width of $w_{t}$, and the tooth depth of $d$.

Fig. 3. (Color online) (a) Transmission of the single tooth-shaped MIM waveguide compared with a straight MIM slit waveguide. The width of the waveguide is $w=50 \mathrm{~nm}$, and the tooth width and depth are respectively $w_{t}=50 \mathrm{~nm}$ and $d=100 \mathrm{~nm}$. The contour profiles of field $H_{y}$ of the toothshaped waveguide at different wavelengths of (b) $\lambda=510 \mathrm{~nm}$, (c) $\lambda=783 \mathrm{~nm}$, and (d) $\lambda=1550 \mathrm{~nm}$. In the FDTD simulation, we used the tabulation of the optical constants of silver given in Ref. 22.

Fig. 4. (Color online) (a) Transmission spectra of the waveguide filters with various tooth widths of $w_{t}$, at a fixed tooth depth of $d=100 \mathrm{~nm}$ and the slit width of $w=50 \mathrm{~nm}$. (b) The wavelength of the trough vs. the tooth width of $w_{t}$.

Fig. 5. (Color online) (a) Transmission spectra of the waveguide filters with different tooth depths of $d$, and with a given tooth width of $w_{t}=50 \mathrm{~nm}$ and the slit width of $w=50 \mathrm{~nm}$. (b) The wavelength of the trough vs. the tooth depth of $d$ with $w_{t}=15 \mathrm{~nm}, w_{t}=30 \mathrm{~nm}$ and $w_{t}=50 \mathrm{~nm}$. 